\title{
Das neue Strahlenschutzrecht - Der Medizinphysik-Experte
}

\section{Einleitung}

Die Richtlinie 2013/59/EURATOM vom 5. Dezember 2013 soll als europarechtliche Vorgabe den Strahlenschutz am Arbeitsplatz und für die Bevölkerung sowie den medizinischen Strahlenschutz innerhalb der europäischen Union verbessern. Die Richtlinie basiert auf den letzten wissenschaftlichen Erkenntnissen und soll einen umfassenden Schutz vor ionisierender Strahlung gewährleisten. Sie enthält u. a. Vorgaben für medizinische Früherkennungsuntersuchungen mit Röntgenstrahlung. Hierdurch sollen nicht erforderliche Röntgenuntersuchungen vermieden werden. Die fünf weiteren Richtlinien 89/618/Euratom, 90/641/Euratom, 96/29/Euratom, 97/43/Euratom und 2003/ 122/Euratom auf diesem Regelungsbereich wurden mit Ende der Umsetzungsfrist zum 6. Februar 2018 aufgehoben. Die Richtlinie musste bis zum Ende dieser Frist in nationales Recht umgesetzt werden.

Am 27. Juni 2017 ist das Gesetz zur Neuordnung des Rechts zum Schutz vor der schädlichen Wirkung ionisierender Strahlung (Strahlenschutzgesetz - StrISchG) verkündet worden. Das StrlSchG ist mit Wirkung zum 01.10.2017 und 31.12.2018 in Kraft getreten und setzt die neue Euratom-Richtlinie in deutsches Recht um. Dabei werden bisherige Regelungen aus der Strahlenschutzverordnung, der Röntgenverordnung und dem Strahlenschutzvorsorgegesetz zusammengeführt und die Vorgaben an den letzten Stand des wissenschaftlichen Fortschritts angepasst. Hierdurch werden auch wesentliche Bereiche in der Radiologie und Strahlentherapie neu geregelt.

Aus diesem Anlass sollen ausgewählte Bereiche der neuen Rechtsmaterie nach individueller Auswahl und möglicherweise aktuellen Begebenheiten - in der Frequenz nicht zwangsläufig regelmäßig - behandelt werden. Dieser erste Teil befasst sich nun mit der zusätzlichen Einbeziehung von Medizinphysik-Experten nach den neuen rechtlichen Vorgaben.

\section{Begriffsbestimmung}

Nach §5 Abs. 24 StrlSchG ist ein Medizinphysik-Experte (nachfolgend: MPE) eine Person mit Masterabschluss in medizinischer Physik oder eine in medizinischer Physik gleichwertig ausgebildete Person mit Hochschulabschluss, die jeweils die erforderliche Fachkunde im Strahlenschutz besitzt.

Für die Strahlentherapie und Nuklearmedizin wurden aufgrund der durch die Richtlinien 96/29/Euratom und 97/43/Euratom aufgestellten Anforderungen bereits in der Vergangenheit Lösungen für eine Einbindung von Medizinphysik-Experten etabliert und in der Richtlinie Strahlenschutz in der Medizin mit Wirkung ab dem 1. November 2011 konkretisiert. Für diese Bereiche sind in Anlage A 2 der Richtlinie die erforderliche Fachkunde, Ausbildung, praktische Erfahrung (Sachkunde), Kurse im Strahlenschutz, Fachkundenachweis, Aufgaben und Qualitätsniveau für MPEs geregelt.

Für die Hinzuziehung von MPEs bei medizinisch-radiologischen Tätigkeiten hat die Strahlenschutzkommission in der Sitzung am 25./26. September 2017 auf Anfrage des Bundesministeriums für Umwelt, Naturschutz, Bau und Reaktorsicherheit (BMUB) eine Empfehlung verabschiedet. Diese Empfehlung beinhaltete insbesondere Angaben zum Grad der Hinzuziehung sowie allgemeine und zusätzliche Aufgaben der MPEs auf diesem Gebiet und weiteren Themenfeldern wie den Qualifikationsvoraussetzungen für den MPE in der Röntgendiagnostik; diese sind adäquate Ausbildung, praktische Erfahrung (Sachkunde), Kurse im Strahlenschutz und regelmäßige Aktualisierung der Fachkunde.

\section{Tätigkeitsfeld}

MPEs sind bei einer Behandlung und Untersuchung mit radioaktiven Stoffen oder ionisierender Strahlung zur engen Mitarbeit bzw. Beratung hinzuziehen. Die konkreten Anforderungen zur Mitarbeit und Beratung durch die MPEs werden auf Verordnungs-

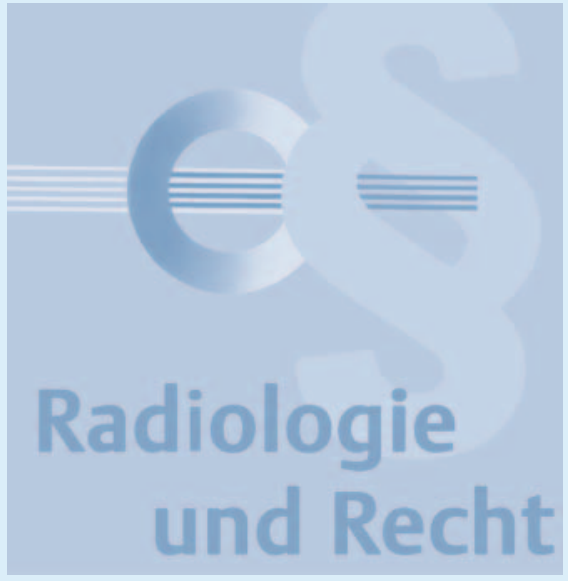

ebene geregelt. Hierzu wurde vornehmlich die Verordnung zur weiteren Modernisierung des Strahlenschutzrechts vom 29. November 2018 erlassen. Durch diese Verordnung sind die alte Strahlenschutzverordnung sowie die Röntgenverordnung zum 31. Dezember 2018 außer Kraft getreten. Gleichzeitig ist insbesondere die neue Strahlenschutzverordnung (StrlSchVO) zum selben Datum in Kraft getreten.

Die StrISchVO definiert die Hinzuziehung und den Aufgabenbereich des MPE in den $\S \S 131$ und 132. Gemäß $\S 131$ Abs. 1 StrISchVO hat der Strahlenschutzverantwortliche dafür zu sorgen, dass bei einer Behandlung mit radioaktiven Stoffen oder ionisierender Strahlung, der ein individueller Bestrahlungsplan zugrunde liegt, ein MPE zur engen Mitarbeit bei der Festlegung des Bestrahlungsplans und der Durchführung der Behandlung hinzugezogen wird. §131 Abs. 2 StrlSchVO konkretisiert die Einsatzgebiete des MPE wie folgt:

- standardisierte Behandlungen mit radioaktiven Stoffen oder ionisierender Strahlung,

- Untersuchungen mit offenen radioaktiven Stoffen,

- Untersuchungen mit ionisierender Strahlung, die mit einem Computertomografen oder mit Geräten zur dreidimensionalen Bildgebung von Objekten mit niedrigem Röntgenkontrast durchgeführt werden mit Ausnahme der Tomosynthese, und

- Interventionen, bei denen die Röntgeneinrichtungen zur Durchleuchtung eingesetzt werden und die mit einer erheblichen Exposition verbunden sind. 
Es obliegt daher zunächst dem Strahlenschutzverantwortlichen, die Verfahrensabläufe durch einen MPE sicherzustellen. Strahlenschutzverantwortlicher ist, wer einer Genehmigung zum Betrieb von Röntgeneinrichtungen oder Störstrahlern bedarf oder wer eine Anzeige zum Betrieb einer Röntgeneinrichtung zu erstatten hat (vgl. §69 Abs. 1 StrlSchG). In Unternehmen oder Gemeinschaftspraxen, mithin bei juristischen Personen oder rechtsfähigen Personengesellschaften, ist Strahlenschutzverantwortlicher die zur Vertretung der juristischen Person oder der rechtsfähigen Personengesellschaft berechtigte Person, z. B. der Geschäftsführer bzw. die Gesellschafter, unabhängig von ihrer Fachkunde im Strahlenschutz. Besteht das vertretungsberechtigte Organ aus mehreren Mitgliedern oder sind bei nicht rechtsfähigen Personenvereinigungen mehrere vertretungsberechtigte Personen vorhanden, so ist der zuständigen Behörde mitzuteilen, welche dieser Personen die Aufgaben des Strahlenschutzverantwortlichen wahrnimmt (vgl. §69 Abs. 2 StrlSchG).

$\S 132$ StrlSchVO regelt den Aufgabenbereich des MPE. Danach hat der Strahlenschutzverantwortliche dafür zu sorgen, dass ein MPE, wenn er nach $\S 131$ hinzuzuziehen ist, die Verantwortung für die Dosimetrie von Personen, an denen radioaktive Stoffe oder ionisierende Strahlung angewendet werden, übernimmt und insbesondere bei der Wahrnehmung der Optimierung des Strahlenschutzes und folgender Aufgaben mitwirkt:

1. Qualitätssicherung bei der Planung und Durchführung von Anwendungen radioaktiver Stoffe oder ionisierender Strahlung am Menschen einschließlich der physikalisch-technischen Qualitätssicherung,

2. Auswahl der einzusetzenden Ausrüstungen, Geräte und Vorrichtungen,

3. Überwachung der Exposition von Personen, an denen radioaktive Stoffe oder ionisierende Strahlung angewendet werden,

4. Überwachung der Einhaltung der diagnostischen Referenzwerte,

5. Untersuchung von Vorkommnissen,

6. Durchführung der Risikoanalyse für Behandlungen und

7. Unterweisung und Einweisung der bei der Anwendung tätigen Personen.
Zwar wird durch die Regelung dem MPE nun ausdrücklich die Verantwortung im Bereich des Strahlenschutzes zugewiesen. Vorrangig obliegt es jedoch dem Strahlenschutzverantwortlichen dafür Sorge zu tragen, dass die Einhaltung der Strahlenschutzvorschriften gewährleistet wird. Er ist einerseits verpflichtet, die Strahlenschutzbeauftragten zu bestimmen, die über die notwendige Fachkunde im Strahlenschutz verfügen, andererseits durch die erweiterten Vorgaben der Verordnung, die MPEs dazu anzuhalten, Verantwortung in den definierten Bereichen zu übernehmen. Der Strahlenschutzverantwortliche verpflichtet sich, eine ausreichende Anzahl von MPEs anzustellen, um die Tätigkeiten, u. a. Dosisabschätzungen, Messung und Rechnung von Strahlenexposition, Prüfung auf Einhaltung der Therapieprotokolle, technische Anleitung der Mitarbeiter etc., zu erfüllen. §44 Abs. 2 Satz 1 StrISchVO normiert, dass der Strahlenschutzverantwortliche seine Pflichten sowie die Pflichten seiner jeweiligen Strahlenschutzbeauftragten, MPEs und sonst unter seiner Verantwortung tätigen Personen vertraglich eindeutig gegeneinander abzugrenzen hat. „Verantwortung zu übernehmen“ im Sinne des $§ 132$ StrlSchVO bedeutet daher zunächst nur, dass der Strahlenschutzverantwortliche die definierten Aufgabenbereiche an die MPEs vertraglich zu übertragen hat und diese dazu anhalten muss, die Aufgaben pflichtgemäß zu erfüllen.

\section{Personalbedarf}

Die Richtlinie Strahlenschutz in der Medizin enthält für Anwendungen in der Strahlentherapie und Nuklearmedizin unter Ziffer 2.1.2. Personalschlüssel als Anhaltszahlen für die Ermittlung des Personalbedarfs an MPEs, anhand derer der eigene Personalbedarf grundsätzlich selbst zu ermitteln ist.

Der Vorstand der Arbeitsgemeinschaft Physik und Technik (APT) der Deutschen Röntgengesellschaft hat unter Zugrundelegung des Berichts Nummer 21 („Empfehlungen zum Personalbedarf in der Medizinischen Strahlenphysik") der Deutschen Gesellschaft für medizinische Physik Personalschlüssel für den zukünftigen Bedarf an MPEs in der Radiologie erstellt und in einem Positionspapier veröffentlicht (RöFo 2014,
S. 419-422), auf das auch das BMU verweist. Hiernach ergibt sich beispielsweise für einen Computertomografen ein Bedarf von 0,06 Vollzeitkräften MPEs. Aus dieser Berechnung folgt ein Gesamtbedarf von 280 MPEs in Deutschland, während die Anzahl für 2014 mit lediglich 100 beziffert wurde.

Bei Betrieb von vier Herzkatheteranlagen, zwei Interventionsplätzen in der Radiologie, ein Hybrid-OP, fünf CT und einem intraoperativen CT ergäbe sich ein Bedarf von 0,92 Stellen. Demnach wäre ab dieser Größenordnung eines Betriebes die Beschäftigung eines eigenen MPE als Vollkraft sinnvoll. Der Vorstand der APT empfiehlt bei entsprechendem Bedarf den Einsatz von hauptamtlichen MPEs, die im Rahmen von Konsultationsverträgen für kleinere Betriebe zur Verfügung stehen könnten. Diese Vorgehensweise könne die flächendeckende Versorgung mit MPEs sicherstellen, ohne dass jeder Betrieb einen eigenen MPE beschäftigen müsse und es zu einer übermäßigen finanziellen Belastung im deutschen Gesundheitswesen käme. Außerdem könnten Kliniken zur medizinphysikalischen Versorgung kooperieren. Mittlere und kleinere Praxen mit geringem Bedarf könnten auch Konsilverträge mit freiberuflichen oder nebenberuflichen MPEs abschließen.

\section{Haftung}

Eine mögliche Haftung des MPE kann allgemein nur skizziert werden, konkret hängt sie von den Bedingungen des Einzelfalls ab. Grundsätzlich erfolgt die Bestellung zum MPE nicht öffentlich-rechtlich durch die Behörde, sondern privatrechtlich, indem der Strahlenschutzverantwortliche mit dem MPE einen Arbeits- bzw. Dienstvertrag abschließt. Der Strahlenschutzverantwortliche hat die auf den MPE zu übertragenen Aufgaben („Verantwortlichkeiten“) klar zu definieren. Im Rahmen des begründeten Anstellungsverhältnisses hat der Strahlenschutzverantwortliche dafür Sorge zu tragen, dass der MPE seine Aufgaben entsprechend der Vorgaben des Strahlenschutzrechts erfüllt. Der MPE handelt als sein Erfüllungsgehilfe im Sinne des $§ 278$ BGB. Erfüllungsgehilfe ist derjenige, wer für einen Dritten (hier Strahlenschutzverantwortlicher) bei einer diesem obliegen- 
den Verbindlichkeit oder Verpflichtung als seine Hilfsperson tätig wird. Der Strahlenschutzverantwortliche hat ein Verschulden der Personen, deren er sich zur Erfüllung seiner Verbindlichkeit bedient, in gleichem Umfang zu vertreten wie eigenes Verschulden.

Der MPE haftet vertraglich gegenüber seinem Arbeitgeber im Innenverhältnis, sofern er gegen seine vertraglichen Pflichten verstößt, durch den Pflichtverstoß ein Schaden verursacht wird und Pflichtverstoß und Schaden vorsätzlich oder grob fahrlässig herbeigeführt wurden.

Der MPE müsste für eine Haftung zunächst gegen eine vertragliche Haupt- oder Nebenpflicht verstoßen. Hierbei ist zu berücksichtigen, dass die technische Durchführung bei der Anwendung ionisierender Strahlung und radioaktiver Stoffe am Menschen durch MPEs nur erfolgt, wenn sie unter ständiger Aufsicht und Verantwortung eines approbierten (Zahn-) Arztes stehen, der die erforderliche Fachkunde im Strahlenschutz besitzt (vgl. §145 Abs. 2 Nr. 6 StrlSchVO). Die Arbeit des MPE erfolgt daher ausschließlich in Zusammenarbeit (und unter Kontrolle) eines fachkundigen Arztes. Ein alleiniges und eigenverantwortliches Tätigwerden des MPE, aus dem ein (alleiniger) Pflichtenverstoß hergeleitet werden könnte, der zu einer Gesundheitsschädigung am Menschen führt und einen Schadensersatzanspruch begründen könnte, dürfte in der Regel nicht in Betracht kommen.
Jedenfalls gilt, dass einem Patienten beispielsweise ein Schmerzensgeldanspruch nur zusteht, wenn nachweisbar ist, dass er durch den MPE körperlich misshandelt oder an der Gesundheit beschädigt wurde. Hier müsste der MPE selbst das schädigende Ereignis schuldhaft herbeigeführt haben, indem er bei der Wahrnehmung seiner Aufgaben, beispielsweise der Qualitätssicherung oder der Auswahl der einzusetzenden Geräte, den Pflichtenverstoß begangen hat. Ferner müsste die Geringfügigkeitsschwelle überschritten worden sein, was bei unbedeutenden Eingriffen, die das Wohlbefinden des Verletzten nur kurzfristig und unerheblich beeinträchtigen, nicht der Fall ist (vgl. OLG Hamm, Urteil vom 27.06.2017 - Az.: 26 U 109/13).

Sofern ein vertraglicher Pflichtenverstoß zu bejahen wäre, der im Kausalzusammenhang mit einem entstandenen Schaden steht, haftet vertraglich im Außenverhältnis grundsätzlich der Arbeitgeber, der den Arbeitnehmer bei der Erfüllung seiner Obliegenheiten als Erfüllungsgehilfen hinzugezogen hat. Der Arbeitgeber kann dann im Innenverhältnis den Arbeitnehmer, den MPE, in Regress nehmen, sofern dieser den Pflichtenverstoß vorsätzlich oder grob fahrlässig herbeigeführt hat. Handelte er leicht fahrlässig, ist seine Haftung ausgeschlossen.

Ob sich der MPE durch die pflichtwidrige Wahrnehmung seiner Aufgaben strafbar macht, lässt sich nicht pauschal beantworten. Die Erfüllung des Straftatbestandes der Körperverletzung im Sinne der $\S \S 223$, 224 StGB, ob fahrlässig oder vorsätzlich, hängt auch von dem Zusammenwirken mit dem jeweiligen fachkundigen Arzt ab, der die Kontrolle und die Verantwortung über den MPE hat. Auch ist der Umfang der Einwilligung des Patienten und die vorhergegangene Aufklärung maßgeblich, da diese ggf. die Tatbestandserfüllung bereits entfallen lässt.

Gleiches gilt hinsichtlich der Beurteilung der Frage, ob der MPE durch sein Handeln auch andere strahlenschutzrechtlich relevanten Strafvorschriften verstoßen kann, beispielsweise $\S 325$ a StGB, Verursachen von Lärm, Erschütterungen und nichtionisierende Strahlen, oder $\S 328$, Unerlaubter Umgang mit radioaktiven Stoffen und anderen gefährlichen Stoffen und Güter.

Prof. Dr. Peter Wigge

Rechtsanwalt

Fachanwalt für Medizinrecht

Tilmann Kirsch

Rechtsanwalt

Rechtsanwälte Wigge

Großer Burstah 42

20457 Hamburg

Telefon: (040) 3398705 - 90

Telefax: (040) 3398705 - 99

Internet: www.ra-wigge.de

E-Mail: kanzlei@ra-wigge.de 